\title{
Blending in the extended finite element method by discontinuous Galerkin and assumed strain methods
}

\author{
Robert Gracie, Hongwu Wang and Ted Belytschko*,† \\ Department of Mechanical Engineering, Northwestern University, 2145 Sheridan Road, Evanston, \\ IL 60208-3111, U.S.A.
}

\begin{abstract}
SUMMARY
In the extended finite element method (XFEM), errors are caused by parasitic terms in the approximation space of the blending elements at the edge of the enriched subdomain. A discontinuous Galerkin (DG) formulation is developed, which circumvents this source of error. A patch-based version of the DG formulation is developed, which decomposes the domain into enriched and unenriched subdomains. Continuity between patches is enforced with an internal penalty method. An element-based form is also developed, where each element is considered a patch. The patch-based DG is shown to have similar accuracy to the element-based DG for a given discretization but requires significantly fewer degrees of freedom. The method is applied to material interfaces, cracks and dislocation problems. For the dislocations, a contour integral form of the internal forces that only requires integration over the patch boundaries is developed. A previously developed assumed strain (AS) method is also developed further and compared with the DG method for weak discontinuities and linear elastic cracks. The DG method is shown to be significantly more accurate than the standard XFEM for a given element size and to converge optimally, even where the standard XFEM does not. The accuracy of the DG method is similar to that of the AS method but requires less application-specific coding. Copyright (C) 2007 John Wiley \& Sons, Ltd.
\end{abstract}

Received 20 July 2007; Revised 7 September 2007; Accepted 14 September 2007

KEY WORDS: discontinuous Galerkin, DG; extended finite element method, XFEM; assumed strain, AS; partition of unity, PU; blending elements; dislocations; cracks; interfaces

\footnotetext{
${ }^{*}$ Correspondence to: Ted Belytschko, Department of Mechanical Engineering, Northwestern University, 2145 Sheridan Road, Evanston, IL 60208-3111, U.S.A.

†E-mail: tedbelytschko@northwestern.edu

Contract/grant sponsor: Publishing Arts Research Council; contract/grant number: 98-1846389

Contract/grant sponsor: Office of Naval Research; contract/grant number: N00014-06-1-380

Contract/grant sponsor: Army Research Office; contract/grant number: W911NF-05-1-0049

Contract/grant sponsor: Natural Sciences and Engineering Research Council
} 


\section{R. GRACIE, H. WANG AND T. BELYTSCHKO}

\section{INTRODUCTION}

Enriched finite element methods (FEMs), such as the extended finite element method (XFEM) of Belytschko and Black [1] and Moës et al. [2] and the global partition of unity method (PUM) of Melenk and Babuška [3], are a powerful way of augmenting standard finite element (FE) approximations using known information about the solution of the problem. It is generally desirable to limit the enrichment to the vicinity of the feature in order to reduce the number of unknowns and improve the conditioning of the system of equations.

Local enrichments have been applied successfully to numerous problems. Recent applications include dynamic crack and shear band propagation, Song et al. [4]; cohesive fracture, Asferg et al. [5]; polycrystals and grain boundaries, Simone et al. [6]; and dislocations, Ventura et al. [7] and Gracie et al. [8]. The method is more accurate than the standard FEM; however, when enrichment is applied to problems with singular fields, the reported results often show the same convergence rate as the standard FEM, which is suboptimal. This degradation of the convergence rate is attributed in part to parasitic terms in the approximation space that arises in the blending elements. A blending element is an element where some but not all of the nodes of the element are enriched. Sukumar et al. [9] showed that parasitic terms in the blending elements limit the accuracy of local PUM methods. Chessa et al. [10] eliminated the parasitic terms by applying an assumed strain (AS) method in the blending elements. The larger approximation space of higher-order spectral elements has also been shown to improve the accuracy in the blending elements [11].

Here, we develop a new method for circumventing the spurious behaviour of the blending elements through a discontinuous Galerkin (DG) approach. We will refer to the proposed method as DG-XFEM. The DG method was introduced by Reed and Hill [12] to solve neutron transport problems. Since then many variants of the original method have evolved, such as the method of Bassi and Rebay [13], the local DG method of Cockburn and Shu [14], the discontinuous hp method of Baumann and Oden [15] and the internal penalty (IP) method of Wheeler [16] and Arnold [17]. Recently, Arnold et al. [18] have presented a unified framework for nine of the most common DG methods. An introduction to DG methods for solids is given by Pfeiffer [19].

In the proposed DG-XFEM method, the domain is decomposed into patches where enrichments are to be added. Each patch is discretized independently. Enrichments are applied over entire patches but not over the entire domain. As a result the enrichment is local but does not require blending elements. Continuity between the patches is enforced in a weak sense using the IP method $[16,17]$. The IP method is stable and consistent and has been shown to converge optimally in both the $H^{1}$ and $L^{2}$ norms for the Poisson equation [18]. This approach is easy to apply and yields very accurate solutions.

We also develop an AS framework similar to Chessa et al. [10], denoted as AS-XFEM, for elastic cracks. The AS approximation is designed to eliminate the parasitic terms in the strain approximation, leading to improved accuracy and optimal convergence rates. The primary drawback of the AS method is the difficulty in constructing the basis functions for the AS approximation. The functions must be linearly independent and span the space of the parasitic terms and furthermore must be constructed for each choice of enrichment. In addition, the method entails the identification of the blending elements and special formulations for these elements. Thus, it lacks the generality and ease of implementation of DG-XFEM. We will compare the AS-XFEM method with the DG-XFEM method for cracks. 
The DG-XFEM presented here is similar to the discontinuous enrichment method (DEM) of Farhat et al. [20], but in the latter, Lagrange multipliers are used to weakly enforce continuity between enriched and unenriched subdomains. An advantage of DEM is that the enrichment degrees of freedom can be statically condensed on the element level, so the only additional degrees of freedom stem from the Lagrange multipliers. Duarte et al. [21] have used a DG method with a PUM enrichment for the singularities that arise in a one-dimensional elastodynamics problem. Laborde et al. [22] improved XFEM for linear elastic cracks with domain decomposition and pointwise matching between the enriched and unenriched subdomains. The resulting approximation is discontinuous and is reported to be slightly superconvergent, $O\left(h^{1.1}\right)$.

In the following section, we review the XFEM approximation and blending elements. In Section 3, the DG formulation for XFEM is described and in Section 4 we briefly recall the AS formulation. The application of the AS method for elastic cracks is presented in Section 5. In Section 6 the simplified discrete DG-XFEM equations for dislocation modelling are derived. Several examples, covering a wide range of applications, are presented in Section 7. Section 8 gives a discussion of our results and our conclusions.

\section{BLENDING ELEMENTS}

In this section, we describe the standard XFEM formulation and define blending elements. The XFEM approximation has the form

$$
\mathbf{u}=\mathbf{u}^{C}+\mathbf{u}^{E}
$$

where $\mathbf{u}^{C}$ is the standard FEM approximation and $\mathbf{u}^{E}$ is the enrichment. The standard part of the approximation is

$$
\mathbf{u}^{C}=\sum_{I \in S} N_{I}(\mathbf{x}) \mathbf{u}_{I}
$$

where $S$ is the set of all nodes, $N_{I}$ are the shape functions and $\mathbf{u}_{I}$ are the nodal unknowns. The augmentation of the standard FEM, known as the enriched part of the approximation, is

$$
\mathbf{u}^{E}=\sum_{\tau=1}^{n_{\mathrm{enr}}} \sum_{J \in S^{\tau}} N_{J}(\mathbf{x}) \Psi^{\tau}(x) \mathbf{a}_{J}^{\tau}
$$

where $n_{\mathrm{enr}}$ is the number of enrichments, $\Psi^{\tau}(\mathbf{x})$ are enrichment functions, $S^{\tau}$ is the set of nodes enriched by $\Psi^{\tau}(\mathbf{x})$ and $\mathbf{a}_{J}^{\tau}$ are the unknowns associated with node $J$ for enrichment function $\tau$. We will use boldface to denote tensors and matrices and a superscript $T$ to denote the transpose operator.

A blending element of enrichment function $\tau$ is denoted as $\Omega_{\tau, e}^{B}$ and is defined as an element with nodes $S^{e}$ for which $S^{\tau} \cap S^{e} \neq S^{e}$ and $S^{\tau} \cap S^{e} \neq \emptyset$. Let $\Omega_{\tau}^{B}=\bigcup_{\forall e} \Omega_{\tau, e}^{B}$ be the blending domain of enrichment $\tau$. Since only some nodes of the blending elements are enriched, the shape functions that premultiply the enrichment functions $\Psi^{\tau}$ in (3) do not satisfy the PU property, i.e.

$$
\sum_{I \in S^{\tau}} N_{I}(\mathbf{x}) \neq 1 \quad \text { for } \mathbf{x} \in \Omega_{\tau}^{B}
$$




\section{R. GRACIE, H. WANG AND T. BELYTSCHKO}

As a result, the enrichment function cannot be reproduced in $\Omega_{\tau}^{B}$ by the enriched approximation, $\mathbf{u}^{E}$. Furthermore, a linear function cannot be reproduced in the blending elements when $\mathbf{a}_{J}^{\tau} \neq 0$ for some node $J$ in the blending element.

Failure to satisfy the PU property can lead to large errors in the blending elements and poor global convergence. Chessa et al. [10] showed that for the ramp enrichment $(\Psi(x)=\langle x\rangle)$, the error in the blending elements scales linearly with element size, whereas it scales quadratically in fully enriched and unenriched elements.

When XFEM is applied to linear elastic fracture mechanics with three-node triangular elements, the parasitic terms in the approximation space of the blending elements lead to poorly satisfied traction boundary conditions along the crack faces. We will study the case of a crack subjected to Mode I loading in Section 7.3.1, but here we indicate the extent to which the standard XFEM does not satisfy the traction-free boundary conditions, see Figure 7(b). The normal stress should be close to zero (the natural boundary condition); however, it can be seen to deviate substantially from zero. While a natural boundary condition will not be satisfied exactly in any FE solution, the error here is too large. We observe that the parasitic terms in the blending elements appear to affect the accuracy of the solution in all elements that are enriched.

\section{DISCONTINUOUS GALERKIN METHOD}

In this section we describe XFEM with a DG formulation, DG-XFEM, which eliminates the need for blending elements. In this approach the domain is decomposed into a set of non-overlapping patches. Enrichments are then applied over these patches and continuity is enforced on the edges of the patches by an IP method.

Consider the domain $\Omega$ with boundary $\Gamma$. On a section of the boundary $\Gamma_{u}$ the displacement boundary conditions are $\mathbf{u}=\overline{\mathbf{u}}$ and on $\Gamma_{t}=\Gamma / \Gamma_{u}$ the tractions are $\mathbf{t}=\overline{\mathbf{t}}$. The domain $\Omega$ is partitioned into $n_{p}$ non-overlapping patches $\Omega_{\beta}^{P}, \beta=1$ to $n_{p}$. Each patch $\beta$ is enriched by the enrichment functions, $\Psi^{\alpha \beta} ; \alpha=1$ to $n_{\beta}^{\mathrm{enr}}$, where $n_{\beta}^{\mathrm{enr}}$ is the number of enrichment functions for patch $\beta$. When patch $\beta$ is not enriched by any enrichment functions $n_{\beta}^{\mathrm{enr}}=0$. The boundary of patch $\beta$ is denoted by $\partial \Omega_{\beta}^{P}$. Let $\partial \Omega_{\beta \theta}=\partial \Omega_{\beta}^{P} \cap \partial \Omega_{\theta}^{P}$ be the intersection of the boundaries of patches $\beta$ and $\theta$. Note that several patches may be enriched by the same enrichment function; this will occur whenever the domains of two enrichment functions overlap. We have chosen to denote the enrichment functions of each patch as separate functions because it is convenient for the discretization of the governing equations.

An example of the decomposition of a domain into patches is shown in Figure 1; it illustrates a domain, $\Omega$; enrichments $\Psi^{1}$ and $\Psi^{2}$ are applied over subdomains $\Omega_{1}^{E}$ and $\Omega_{2}^{E}$, respectively. The boundaries of $\Omega_{1}^{E}$ and $\Omega_{2}^{E}$ decompose the domain, $\Omega$, into four patches. Patches $\Omega_{1}^{P}$ and $\Omega_{2}^{P}$ are enriched by $\Psi^{1}$ and $\Psi^{2}$, respectively. Patch $\Omega_{3}^{P}$ is enriched by both $\Psi^{1}$ and $\Psi^{2}$ and patch $\Omega_{4}^{P}$ is unenriched.

The displacement approximation on patch $\beta$ is

$$
\mathbf{u}^{\beta}(\mathbf{x})=\sum_{I \in \mathscr{S}_{\beta}^{P}} N_{I}(\mathbf{x})\left(\mathbf{u}_{I}^{\beta}+\sum_{\alpha=1}^{n_{\beta}^{\mathrm{enr}}} \Psi^{\alpha \beta}(\mathbf{x}) \mathbf{a}_{I}^{\alpha \beta}\right)
$$



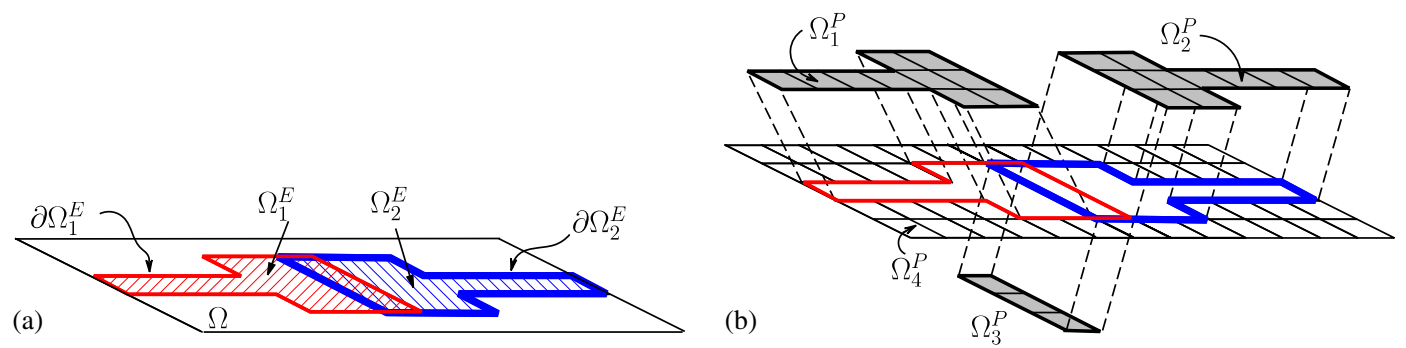

Figure 1. (a) Illustration of a domain with two overlapping enrichment domains and (b) decomposition of the domain in (a) into four patches defined by the boundaries of the enrichment domain.

where $\mathscr{S}_{\beta}^{P}$ is the set of nodes in patch $\beta$. When patch $\beta$ is not enriched by any enrichment functions $n_{\beta}^{\mathrm{enr}}=0$ and (5) simplifies to the standard FEM approximation. We note that the PU property is satisfied everywhere on each patch. We define the displacement jump across $\partial \Omega_{\beta \theta}$ by

$$
\left[\left|\mathbf{u}^{\beta \theta}\right|\right]=\frac{1}{2}\left(\mathbf{u}^{\beta}-\mathbf{u}^{\theta}\right), \quad \beta=1 \ldots n_{p} \text { and } \theta=1 \ldots n_{p}
$$

and the average traction by

$$
\left\langle\mathbf{t}^{\beta \theta}\right\rangle=\frac{1}{2}\left(\mathbf{t}^{\beta}+\mathbf{t}^{\theta}\right), \quad \beta=1 \ldots n_{p} \text { and } \theta=1 \ldots n_{p}
$$

where $\mathbf{u}^{\beta}, \mathbf{t}^{\beta}=\boldsymbol{\sigma}^{\beta} \cdot \mathbf{n}^{\beta}$ and $\mathbf{n}^{\beta}$ are the displacement, traction and normal on the boundary of patch $\beta$ and $\boldsymbol{\sigma}^{\beta}$ is the stress on the boundary of patch $\beta$.

Continuity between patches is enforced in a weak sense by the penalty method of Wheeler [16] and Arnold [17]. The DG-XFEM weak form is: find $\mathbf{u}=\left\{\mathbf{u}^{1}, \mathbf{u}^{2}, \ldots, \mathbf{u}^{n_{P}}\right\}, \mathbf{u}^{\beta} \in \mathscr{U}^{\beta}$ such that

$$
\begin{aligned}
\delta \Pi^{\mathrm{DG}}= & \sum_{\beta=1}^{n_{P}}\left[\int_{\Omega_{\beta}^{P}} \boldsymbol{\varepsilon}\left(\delta \mathbf{v}^{\beta}\right) \cdot \boldsymbol{\sigma}\left(\boldsymbol{\varepsilon}\left(\mathbf{u}^{\beta}\right)\right) \mathrm{d} \Omega+\sum_{\theta>\beta}^{n_{P}}\left(\frac{\alpha}{A} \int_{\partial \Omega_{\beta \theta}}\left[\left|\delta \mathbf{v}^{\theta \beta}\right|\right]\left[\left|\mathbf{u}^{\theta \beta}\right|\right] \mathrm{d} \Gamma\right.\right. \\
& \left.\left.-\mu_{1} \int_{\partial \Omega_{\beta \theta}}\left\langle\delta \mathbf{t}^{\theta \beta}\right\rangle\left[\left|\mathbf{u}^{\theta \beta}\right|\right] \mathrm{d} \Gamma-\mu_{2} \int_{\partial \Omega_{\beta \theta}}\left[\left|\delta \mathbf{v}^{\theta \beta}\right|\right]\left\langle\mathbf{t}^{\theta \beta}\right\rangle \mathrm{d} \Gamma\right)\right]-\delta W^{\mathrm{ext}}=0 \quad \forall \delta \mathbf{v}^{\beta} \in \mathscr{V}^{\beta}
\end{aligned}
$$

where $A$ is a measure of the domain of an element. In the present work we take $\mu_{1}=\mu_{2}=1 ; \alpha$ is a penalty-like constant and the term it multiplies is a stabilization term. This penalty-like parameter is problem dependent. The spaces of admissible trial and test functions are

$$
\begin{aligned}
& \mathscr{U}^{\beta}=\left\{\mathbf{u} \mid \mathbf{u} \in \mathscr{H}^{1}\left(\Omega_{\beta}^{P}\right), \mathbf{u}=\overline{\mathbf{u}} \text { on } \Gamma_{u}\right\} \\
& \mathscr{V}^{\beta}=\left\{\mathbf{u} \mid \mathbf{u} \in \mathscr{H}^{1}\left(\Omega_{\beta}^{P}\right), \mathbf{u}=0 \text { on } \Gamma_{u}\right\}
\end{aligned}
$$

respectively. We, as other authors, have found that $\alpha$ can be much smaller than standard penalty parameters; the specific values used here are presented later.

We adopt the linear elastic stress strain law, which in Voigt notation is

$$
\boldsymbol{\sigma}(\boldsymbol{\varepsilon})=\mathbf{C} \varepsilon
$$




\section{R. GRACIE, H. WANG AND T. BELYTSCHKO}

and the strain-displacement equation

$$
\boldsymbol{\varepsilon}(\mathbf{u})=\vec{\nabla}^{s} \mathbf{u}
$$

where $\vec{\nabla}^{s} \mathbf{u}$ is the symmetric gradient of $\mathbf{u}$.

The discrete equations are obtained by substituting (11), (12) and the displacement approximation for each patch (5) into the weak form (8), which gives

$$
\left(\mathbf{K}^{\mathrm{XFEM}}+\mathbf{K}^{\mathrm{DG}}\right) \mathbf{d}=\mathbf{f}^{\mathrm{ext}}
$$

where $\mathbf{d}^{\top}=\left\{\mathbf{d}_{1}^{1}, \mathbf{d}_{2}^{1}, \ldots, \mathbf{d}_{m_{1}}^{1}, \ldots, \mathbf{d}_{1}^{n_{p}}, \mathbf{d}_{2}^{n_{p}}, \ldots, \mathbf{d}_{m_{n_{p}}}^{n_{p}}\right\}$ is the vector of nodal degrees of freedom and $m_{\beta}$ is the number of nodes in patch $\beta$. The nodal vector for node $I$ of patch $\beta$ is $\left(\mathbf{d}_{I}^{\beta}\right)^{\top}=$ $\left\{\mathbf{u}_{I}^{\beta},\left(\mathbf{a}_{I}^{1}\right)^{\beta}, \ldots,\left(\mathbf{a}_{I}^{n_{\beta}^{\mathrm{enr}}}\right)^{\beta}\right\}$. The stiffnesses are

$$
\begin{aligned}
\mathbf{K}^{\mathrm{XFEM}} & =\sum_{\beta=1}^{n_{p}} \mathbf{K}^{P \beta}, \quad \mathbf{K}_{I J}^{P \beta}=\int_{\Omega_{e}} \mathbf{B}_{I}^{\top} \mathbf{C B}_{J} \mathrm{~d} \Omega, \quad I, J \in \mathscr{S}_{\beta}^{P} \\
\mathbf{K}^{\mathrm{DG}} & =\sum_{\beta=1}^{n_{p}} \sum_{\theta>\beta}^{n_{p}}\left[\mathbf{K}_{\alpha}^{\beta \theta}-\mathbf{K}_{\mu}^{\beta \theta}-\left(\mathbf{K}_{\mu}^{\beta \theta}\right)^{\top}\right]
\end{aligned}
$$

where

$$
\begin{aligned}
& \mathbf{K}_{\alpha I J}^{\beta \theta}=\frac{\alpha}{4 A} \int_{\partial \Omega_{\beta \theta}}\left(\overline{\mathbf{N}}_{I J}^{\beta \theta}\right)^{\top} \mathbf{C}_{\overline{\mathbf{N}}_{I J}^{\beta \theta}}^{\beta \theta} \mathrm{d} \Gamma, \quad I \in \mathscr{S}_{\beta}^{P}, \quad J \in \mathscr{S}_{\theta}^{P} \\
& \mathbf{K}_{\mu I J}^{\beta \theta}=\frac{1}{4} \int_{\partial \Omega_{\beta \theta}}\left(\overline{\mathbf{B}}_{I J}^{\beta \theta}\right)^{\top} \mathbf{C} \overline{\mathbf{N}}_{I J}^{\beta \theta} \mathrm{d} \Gamma, \quad I \in \mathscr{S}_{\beta}^{P}, \quad J \in \mathscr{S}_{\theta}^{P}
\end{aligned}
$$

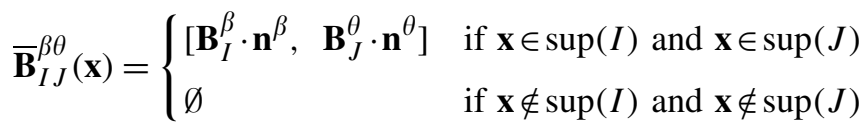

$$
\begin{aligned}
& \overline{\mathbf{N}}_{I J}^{\beta \theta}(\mathbf{x})= \begin{cases}{\left[\mathbf{N}_{I}^{\beta},-\mathbf{N}_{J}^{\theta}\right]} & \text { if } \mathbf{x} \in \sup (I) \text { and } \mathbf{x} \in \sup (J) \\
\varnothing & \text { if } \mathbf{x} \notin \sup (I) \text { and } \mathbf{x} \notin \sup (J)\end{cases}
\end{aligned}
$$

and where $\sup (I)$ denotes the support of node $I$. The nodal matrices $\mathbf{B}_{I}^{\beta}$ and $\mathbf{N}_{I}^{\beta}$ (for node $I$ on patch $\beta$ ) are defined in the standard way such that $\nabla^{s} \mathbf{u}^{\beta}=\sum_{I \in S_{\beta}^{P}} \mathbf{B}_{I}^{\beta} \mathbf{d}_{I}^{\beta}$, and $\mathbf{u}^{\beta}=\sum_{I \in S_{\beta}^{P}} \mathbf{N}_{I}^{\beta} \mathbf{d}_{I}^{\beta}$. $\mathbf{B}_{I}^{\beta}$ and $\mathbf{N}_{I}^{\beta}$ can be decomposed into continuous and enriched parts: $\mathbf{B}_{I}^{\beta}=\left[\mathbf{B}_{I}^{C, \beta}, \mathbf{B}_{I}^{E, \beta}\right]$ and $\mathbf{N}_{I}^{\beta}=$ $\left[\mathbf{N}_{I}^{C, \beta}, \mathbf{N}_{I}^{E, \beta}\right]$, respectively. In two dimensions, the matrices are

$$
\mathbf{B}_{I}^{C, \beta}=\left[\begin{array}{cc}
N_{I, x} & 0 \\
0 & N_{I, y} \\
N_{I, y} & N_{I, x}
\end{array}\right]
$$


BLENDING IN XFEM BY DG AND AS METHODS

$$
\begin{aligned}
\mathbf{B}_{I}^{E, \beta} & =\left[\begin{array}{ccccc}
\left(\Psi^{1 \beta} N_{I}\right)_{, x} & 0 & \ldots & \left(\Psi^{n_{\beta}^{\mathrm{enr}} \beta} N_{I}\right)_{, x} & 0 \\
0 & \left(\Psi^{1 \beta} N_{I}\right)_{, y} & \ldots & 0 & \left(\Psi^{n_{\beta}^{\mathrm{enr}} \beta} N_{I}\right)_{, y} \\
\left(\Psi^{1 \beta} N_{I}\right)_{, y} & \left(\Psi^{1 \beta} N_{I}\right)_{, x} & \ldots & \left(\Psi^{n_{\beta}^{\mathrm{enr}} \beta} N_{I}\right)_{, y} & \left(\Psi^{n_{\beta}^{\mathrm{enr}} \beta} N_{I}\right)_{, x}
\end{array}\right] \\
\mathbf{N}_{I}^{C, \beta} & =\left[\begin{array}{cc}
N_{I} & 0 \\
0 & N_{I}
\end{array}\right] \\
\mathbf{N}_{I}^{E, \beta} & =\left[\begin{array}{ccccc}
\Psi^{1 \beta} N_{I} & 0 & \ldots & \Psi_{\beta}^{n^{\mathrm{enr}} \beta} N_{I} & 0 \\
0 & \Psi^{1 \beta} N_{I} & \ldots & 0 & \Psi^{n_{\beta}^{\mathrm{enr}} \beta} N_{I}
\end{array}\right]
\end{aligned}
$$

where a comma denotes derivatives with respect to the variable that follows. Note that assembly is implied by the summation operators in (14) and (15). $\mathbf{K}^{\mathrm{XFEM}}$ consists of the assembled standard XFEM stiffness matrices of each patch, while $\mathbf{K}^{\mathrm{DG}}$ contains the DG terms that enforce continuity between patches.

We will refer to the above formulation as the patch-based DG-XFEM. We will also consider an element-based DG-XFEM. In the element-based DG-XFEM each element in the mesh is treated as a patch as in the standard DG formulations; hence, the nodes of each element are independent and the DG terms are applied over all element edges.

\subsection{Other possible DG implementations}

For many enrichment functions, it is effective to evaluate the DG terms only for the enriched part of the displacement approximation. The DG terms tend to cause the enrichment to vanish at the edge of the enrichment domain. Although it is less convenient in some applications, we have found that DG-XFEM is more accurate if the constraint terms are applied to the entire field and not just the enrichment. This is similar to what has been reported with the Lagrange multiplier method of Farhat et al. [20, 23].

\section{ASSUMED STRAIN FORMULATION}

The AS method [24] has previously been applied in blending elements to eliminate the parasitic terms in the strain approximation [10]. The AS method is a special case of the Hu-Washizu variational principle, which is known as a multi-field method because separate approximations for

the displacement, $\mathbf{u}$, strain, $\overline{\boldsymbol{\varepsilon}}$, and stress, $\boldsymbol{\sigma}$, are used. The AS-XFEM method approximates the displacement, $\mathbf{u}$, by the standard XFEM approximation (1)-(3). The strain is approximated by

$$
\bar{\varepsilon}=\boldsymbol{\varepsilon}(\mathbf{u})+\varepsilon^{a}
$$

where $\boldsymbol{\varepsilon}(\mathbf{u})=\vec{\nabla}^{s} \mathbf{u}$ and $\boldsymbol{\varepsilon}^{a}$ is the AS given by

$$
\boldsymbol{\varepsilon}^{a}=\sum_{i=1}^{n_{a}^{e}} N_{i}^{\boldsymbol{\varepsilon}}(\mathbf{x}) \boldsymbol{\alpha}_{i}^{e}
$$




\section{R. GRACIE, H. WANG AND T. BELYTSCHKO}

where $N_{i}^{\varepsilon}$ are the AS shape functions, $n_{a}^{e}$ is the number of AS shape functions for element $e$ and $\alpha_{i}^{e}$ are the AS degrees of freedom associated with AS shape function $i$. The superscript $e$ on $\alpha_{i}^{e}$ indicates that these degrees of freedom are element specific since the strain field is not continuous. The coefficients $\alpha_{I}^{e}$ can be eliminated on the element level before the assembly of the global stiffness matrix. The AS shape functions must be orthogonal to a constant field, i.e.

$$
\int_{\Omega^{e}} N_{i}^{\varepsilon}(\mathbf{x}) \mathrm{d} \Omega=0
$$

It has been shown by Stolarski and Belytschko [25] that the AS, $\boldsymbol{\varepsilon}^{a}$, yields no benefits when used to enrich the strain approximation space. It can, however, be used to eliminate parasitic terms from the strain approximation space. Here, the AS shape functions are chosen so as to eliminate the parasitic terms in the blending elements, as described later.

The discrete AS element equations are

$$
\left[\begin{array}{cc}
\mathbf{K}_{d d}^{e} & \mathbf{K}_{\alpha d}^{e \top} \\
\mathbf{K}_{\alpha d}^{e} & \mathbf{K}_{\alpha \alpha}^{e}
\end{array}\right]\left\{\begin{array}{l}
\mathbf{d}^{e} \\
\alpha^{e}
\end{array}\right\}=\left\{\begin{array}{c}
\mathbf{f}^{e} \\
0
\end{array}\right\}
$$

where $\mathbf{d}^{e}$ is a vector of element nodal degrees of freedom, $\boldsymbol{\alpha}^{e}$ is a vector of the AS unknowns, $\mathbf{f}^{e}$ is the standard FEM vector resulting from the body loads, $\mathbf{K}_{d d}^{e}$ is the standard XFEM element stiffness matrix and

$$
\begin{aligned}
\mathbf{K}_{\alpha d}^{e} & =\int_{\Omega_{e}} \overline{\mathbf{N}}^{\varepsilon \top} \mathbf{C B} \mathrm{d} \Omega \\
\mathbf{K}_{\alpha \alpha}^{e} & =\int_{\Omega_{e}} \overline{\mathbf{N}}^{\varepsilon \top} \mathbf{C} \overline{\mathbf{N}}^{\varepsilon} \mathrm{d} \Omega
\end{aligned}
$$

where $\mathbf{B}$ is the standard $B$-matrix resulting from the XFEM approximation, (20) and (21), and $\overline{\mathbf{N}}^{\varepsilon}$ is the matrix containing the AS shape functions. The AS degrees of freedom, $\boldsymbol{\alpha}^{e}$, are evaluated on the element level by static condensation. The choice of the AS shape functions for elastic cracks will be discussed in the following section. See Chessa et al. [10] for a detailed description of the AS method for blending and Belytschko et al. [26] for a discussion on the applications of multi-field methods.

\section{ELASTIC CRACKS}

The enrichments of linear elastic cracks considered here are based on [1,2,27]. The enrichment of the displacement approximation will be presented only in terms of DG-XFEM. The definition of the enrichment functions for AS-XFEM is identical to the standard XFEM. For clarity we will omit the superscripts on the enrichment functions denoting the patches and will only consider a single crack, denoted as $\gamma$.

We define the geometry of the crack using two level set functions, $f_{\gamma}(\mathbf{x})$ and $g_{\gamma}(\mathbf{x})$, as in Stolarska et al. [28] and Belytschko et al. [29]. The crack surface is given by all $\mathbf{x}$ such that $f_{\gamma}(\mathbf{x})=0$ and $g_{\gamma}(\mathbf{x})>0$, while the crack front (or tip in two dimensions) is given by all $\mathbf{x}$ such that $f_{\gamma}(\mathbf{x})=0$ and $g_{\gamma}(\mathbf{x})=0$, see Figure 2 . 


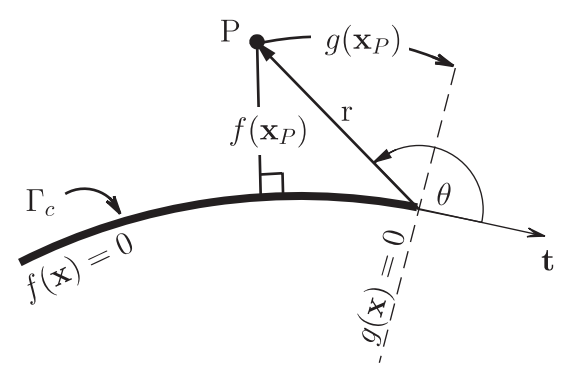

Figure 2. Notation for the representation of a crack $\Gamma_{C}$ by two level set functions $f(\mathbf{x})$ and $g(\mathbf{x})$.

For a given crack, each patch that contains the crack or is near the crack front will be enriched by one of the two types of enrichments. Patches near the crack front are enriched by a set of singular enrichment functions, developed by Fleming et al. [27], based on the near-field asymptotic solution. These functions are often referred to as the branch functions and are given by

$$
\left\{\Psi_{C}\left(r_{\gamma}, \theta_{\gamma}\right)\right\}=\left\{\sqrt{r_{\gamma}} \sin \frac{\theta_{\gamma}}{2}, \sqrt{r_{\gamma}} \cos \frac{\theta_{\gamma}}{2}, \sqrt{r_{\gamma}} \sin \frac{\theta_{\gamma}}{2} \sin \theta_{\gamma}, \sqrt{r_{\gamma}} \cos \frac{\theta_{\gamma}}{2} \sin \theta_{\gamma}\right\}
$$

where $r_{\gamma}$ and $\theta_{\gamma}$ are the polar coordinates of the local coordinate system defined with an origin at the tip of crack $\gamma$ and with basis vectors defined by the unit vectors tangent and normal to the crack at the crack tip, see Figure 2. The discontinuity across the crack surface is introduced into the approximation by the first term in (30).

All patches that are not enriched by (30) but are crossed by crack $\gamma$ are enriched by

$$
\Psi_{H}(\mathbf{x})=H\left(f_{\gamma}(\mathbf{x})\right) H\left(g_{\gamma}(\mathbf{x})\right)
$$

where $H$ is the Heaviside step function given by

$$
H(z)= \begin{cases}1 & \text { if } z>0 \\ 0 & \text { otherwise }\end{cases}
$$

Methods for treating branching cracks and intersecting cracks are given in [29].

\subsection{AS shape functions}

Next we describe the construction of the AS shape functions, $N_{i}^{\varepsilon}$, for the blending elements arising from the enrichment functions (30) for linear elastic cracks. The step function enrichment (31) does not involve any blending elements. For the singular enrichment functions (30) the parasitic terms in the approximation space of the blending elements are spanned by the set

$$
\{\mathscr{P}\}=\left\{\Psi_{C}(\mathbf{x}), \xi_{i} \frac{\partial \Psi_{C}(\mathbf{x})}{\partial x_{j}}\right\}
$$

where $\boldsymbol{\xi}$ are the coordinates of $\mathbf{x}$ in the parent domain. Here we have assumed that the Jacobian between the parent and global coordinate system is constant. Applying (33) to constant stress 


\section{R. GRACIE, H. WANG AND T. BELYTSCHKO}

triangular elements, we have found that the parasitic terms of the strain approximation in the blending element are spanned by a set of functions:

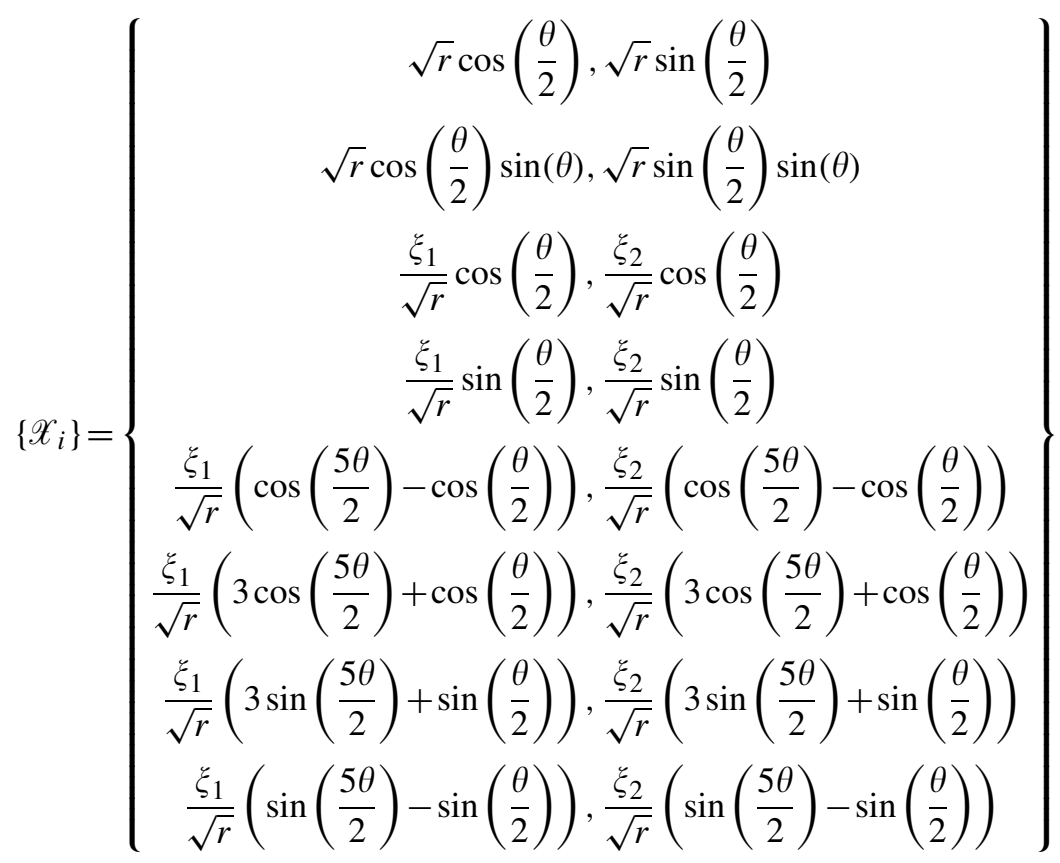

It is desirable to adopt these functions as the AS shape functions used in (25); however, these functions do not satisfy the orthogonality condition (26). The AS shape functions are obtained by orthogonalizing the functions $\mathscr{X}_{i}$, i.e.

$$
N_{i}^{\varepsilon}=\mathscr{X}_{i}-\frac{1}{A_{0}} \int_{\Omega_{e}} \mathscr{X}_{i} \mathrm{~d} \Omega
$$

where $A_{0}$ is the area of the element defined by domain $\Omega_{e}$ and $i$ ranges over all members in $\left\{\mathscr{X}_{i}\right\}$.

\section{EDGE DISLOCATIONS}

In Gracie et al. [8] and Belytschko and Gracie [30], edge dislocations were modelled by a tangential step enrichment function. Ventura et al. [7] treated dislocations by a singular enrichment. We have found that the Peach-Koehler force is most effectively computed by using a combination of singular and tangential step enrichments [31]. The geometry of an edge dislocation $\gamma$ is defined by two level sets, in a manner similar to that for a crack described in the previous section. The glide plane of the dislocation is defined as: all $\mathbf{x}$ such that $f_{\gamma}(\mathbf{x})=0$ and $g_{\gamma}(\mathbf{x})>0$ and the location of the dislocation core is defined as: all $\mathbf{x}$ such that $f_{\gamma}(\mathbf{x})=0$ and $g_{\gamma}(\mathbf{x})=0$. 
All patches in the vicinity of the core of dislocation $\gamma$ are enriched by

$$
\boldsymbol{\Psi}_{\text {core }}(\mathbf{x})=\frac{b_{\gamma}}{2 \pi}\left[\begin{array}{ll}
-\mathbf{e}_{x} \cdot \nabla g & \mathbf{e}_{x} \cdot \nabla f \\
-\mathbf{e}_{y} \cdot \nabla g & \mathbf{e}_{y} \cdot \nabla f
\end{array}\right]\left[\begin{array}{c}
\arctan \frac{f}{g}+\frac{g f}{2(1-v) r^{2}} \\
\frac{2 v-1}{4(1-v)} \ln \left(r^{2}\right)+\frac{f^{2}-g^{2}}{4(1-v) r^{2}}
\end{array}\right]
$$

where $f=f_{\gamma}(\mathbf{x}), g=g_{\gamma}(\mathbf{x}), r^{2}=f^{2}+g^{2}$ and $b_{\gamma}$ is the magnitude of the Burgers vector of dislocation $\gamma$.

All patches that are not in the vicinity of the core of dislocation $\gamma$ but are cut by the glide plane of dislocation $\gamma$ are enriched by

$$
\boldsymbol{\Psi}_{t}(\mathbf{x})=\mathbf{b}_{\gamma} H\left(f_{\gamma}(\mathbf{x})\right) H\left(g_{\gamma}(\mathbf{x})\right)
$$

where $\mathbf{b}_{\gamma}$ is Burgers vector and $H$ is the Heaviside step function given by (32).

\subsection{Application of DG-XFEM to dislocations}

In dislocation modelling the enriched degrees of freedoms in (5) are all prescribed, since the Burgers vectors are given. Hence, we prescribe all enriched degrees of freedom as unity, i.e. $a_{I}^{\alpha \beta}=1$. This introduces a jump across the glide plane with magnitude and direction given by the Burgers vector. Since in the DG-XFEM the PU property is satisfied by the shape functions which premultiply the enrichment functions, (5) can be simplified to

$$
\mathbf{u}^{\beta}(\mathbf{x})=\sum_{I \in \mathscr{S}_{\beta}^{P}} N_{I}(\mathbf{x}) \mathbf{u}_{I}^{\beta}+\mathbf{u}^{E, \beta}(\mathbf{x})=\sum_{I \in \mathscr{S}_{\beta}^{P}} N_{I}(\mathbf{x}) \mathbf{u}_{I}^{\beta}+\sum_{\alpha=1}^{n_{\beta}^{\mathrm{enr}}} \Psi_{\mathrm{core}}^{\alpha \beta}(\mathbf{x})
$$

where $\mathbf{u}^{E, \beta}(\mathbf{x})$ is the enriched part of the approximation of patch $\beta$ which for dislocations is known from the input of the problem. This simplified form of the displacement approximation leads to a simplification of the discrete DG-XFEM equations.

Let $\overline{\mathbf{N}}_{I J}^{C, \beta \theta}$ and $\overline{\mathbf{N}}_{I J}^{E, \beta \theta}$ be the standard continuous and enriched parts of $\overline{\mathbf{N}}_{I J}^{\beta \theta}$, respectively. Also, let $\overline{\mathbf{B}}_{I J}^{C, \beta \theta}$ and $\overline{\mathbf{B}}_{I J}^{E, \beta \theta}$ be the standard continuous and enriched parts of $\overline{\mathbf{B}}_{I J}^{\beta \theta}$, respectively. The discrete equations are

$$
\left(\mathbf{K}^{\mathrm{FE}}+\mathbf{K}_{C C}^{\mathrm{DG}}\right) \mathbf{d}_{C}=\mathbf{f}^{\mathrm{ext}}-\mathbf{f}^{D}-\mathbf{f}^{\mathrm{DG}}
$$

where $\mathbf{K}^{\mathrm{FE}}$ is the standard FE stiffness matrix for an unenriched domain and $\mathbf{d}_{C}$ are the standard continuous degrees of freedom. $\mathbf{K}_{C C}^{\mathrm{DG}}$ is the DG stiffness matrix for an unenriched domain and is given by

$$
\begin{aligned}
\mathbf{K}_{C C}^{\mathrm{DG}} & =\sum_{\beta=1}^{n_{p}} \sum_{\theta>\beta}^{n_{p}}\left[\mathbf{K}_{\alpha}^{C, \beta \theta}-\mathbf{K}_{\mu}^{C, \beta \theta}-\mathbf{K}_{\mu}^{C, \beta \theta \top}\right] \\
\mathbf{K}_{\alpha I J}^{C, \beta \theta} & =\frac{\alpha}{4 A} \int_{\partial \Omega_{\beta \theta}}\left(\overline{\mathbf{N}}_{I J}^{C, \beta \theta}\right)^{\top} \mathbf{C} \overline{\mathbf{N}}_{I J}^{C, \beta \theta} \mathrm{d} \Gamma, \quad I \in \mathscr{S}_{\beta}^{P}, \quad J \in \mathscr{S}_{\theta}^{P} \\
\mathbf{K}_{\mu I J}^{C, \beta \theta} & =\frac{1}{4} \int_{\partial \Omega_{\beta \theta}}\left(\overline{\mathbf{B}}_{I J}^{C, \beta \theta}\right)^{\top} \mathbf{C} \overline{\mathbf{N}}_{I J}^{C, \beta \theta} \mathrm{d} \Gamma, \quad I \in \mathscr{S}_{\beta}^{P}, \quad J \in \mathscr{S}_{\theta}^{P}
\end{aligned}
$$




\section{R. GRACIE, H. WANG AND T. BELYTSCHKO}

The effect of the dislocations appears on the right-hand side of (39) in the nodal force vector, $\mathbf{f}^{\mathrm{D}}$. We define $\left[\left|\mathbf{u}^{E, \beta \theta}\right|\right]$ as the jump in the enriched part of the displacement and $\left\langle\mathbf{t}^{E, \beta \theta}\right\rangle$ as the average traction due to the enrichment. The nodal forces $\mathbf{f}^{\mathrm{DG}}$ are

$$
\mathbf{f}^{\mathrm{DG}}=\sum_{\beta=1}^{n_{p}} \mathbf{f}^{\mathrm{DG}, \beta}, \quad \mathbf{f}^{\mathrm{DG}, \beta}=\sum_{\theta>\beta}^{n_{p}}\left[\mathbf{f}_{\alpha}^{\beta \theta}-\mathbf{f}_{\mu_{1}}^{\beta \theta}-\mathbf{f}_{\mu_{2}}^{\beta \theta}\right]
$$

where

$$
\begin{aligned}
\mathbf{f}_{\alpha I J}^{\beta \theta} & =\frac{\alpha}{4 A} \int_{\partial \Omega_{\beta \theta}}\left(\overline{\mathbf{N}}_{I J}^{C, \beta \theta}\right)^{\top} \mathbf{C}\left[\left|\mathbf{u}^{E, \beta \theta}\right|\right] \mathrm{d} \Gamma, \quad I \in \mathscr{S}_{\beta}^{P}, \quad J \in \mathscr{S}_{\theta}^{P} \\
\mathbf{f}_{\mu_{1} I J}^{\beta \theta} & =\frac{1}{4} \int_{\partial \Omega_{\beta \theta}}\left(\overline{\mathbf{B}}_{I J}^{C, \beta \theta}\right)^{\top} \mathbf{C}\left[\left|\mathbf{u}^{E, \beta \theta}\right|\right] \mathrm{d} \Gamma, \quad I \in \mathscr{S}_{\beta}^{P}, \quad J \in \mathscr{S}_{\theta}^{P} \\
\mathbf{f}_{\mu_{2} I J}^{\beta \theta} & =\frac{1}{4} \int_{\partial \Omega_{\beta \theta}}\left(\overline{\mathbf{N}}_{I J}^{C, \beta \theta}\right)^{\top}\left\langle\mathbf{t}^{E, \beta \theta}\right\rangle \mathrm{d} \Gamma, \quad I \in \mathscr{S}_{\beta}^{P}, \quad J \in \mathscr{S}_{\theta}^{P}
\end{aligned}
$$

The dislocation force from the XFEM approximations is

$$
\mathbf{f}^{D}=\sum_{\beta=1}^{n_{p}} \int_{\Omega_{\beta}^{P}}\left(\mathbf{B}^{C, \beta}\right)^{\top} \boldsymbol{\sigma}^{E, \beta} \mathrm{d} \Omega
$$

where $\boldsymbol{\sigma}^{E, \beta}$ is the part of the stress computed from $\mathbf{u}^{E, \beta}$. If we drop the superscripts in (47) and recall the definition of $\mathbf{B}^{C, \beta}$, (47) can be rewritten for an element as

$$
\left(\mathbf{f}_{i I}^{D}\right)^{e}=\int_{\Omega_{e}} \frac{\partial N_{I}}{\partial x_{j}} \sigma_{j i}^{E} \mathrm{~d} \Omega
$$

where $\Omega_{e}$ is the domain of the element. By Green's theorem, the above right-hand side can be replaced by

$$
\left(\mathbf{f}_{i I}^{D}\right)^{e}=\int_{\partial \Omega_{e}} N_{I} \sigma_{j i}^{E} n_{j}^{e} \mathrm{~d} \Gamma-\int_{\Omega_{e}} N_{I} \sigma_{j i, j}^{E} \mathrm{~d} \Omega
$$

where $\partial \boldsymbol{\Omega}_{e}$ is the boundary of the element and $\mathbf{n}$ is the outward facing normal to the element boundary. When the enrichments are equilibrium solutions, as they are for the dislocations, the last term vanishes.

If we consider a patch $\beta$ consisting of $m_{e}^{\beta}$ elements, then from the above, it follows that

$$
\mathbf{f}_{i I}^{D}=\sum_{\beta=1}^{n_{p}} \sum_{e=1}^{m_{e}^{\beta}} \int_{\partial \Omega_{e}} N_{I} \sigma_{j i}^{E} n_{j}^{e} \mathrm{~d} \Gamma
$$

The contributions of boundaries shared by any pair of elements to the above vanishes. Therefore, the contour integral consists only of the contour around the patch; hence,

$$
\mathbf{f}_{i I}^{D}=\sum_{\beta=1}^{n_{p}} \int_{\partial \Omega_{\beta}^{P}} N_{I} \sigma_{j i}^{E, \beta} n_{j}^{\beta} \mathrm{d} \Gamma
$$


where $\mathbf{n}^{\beta}$ is the outward facing normal to the boundary of patch $\beta$. In the matrix form (with the superscripts) the above can be expressed as

$$
\mathbf{f}_{I}^{D}=\sum_{\beta=1}^{n_{p}} \int_{\partial \Omega_{\beta}^{P}}\left(\mathbf{N}_{I}^{C, \beta}\right)^{\top} \mathbf{t}^{E, \beta} \mathrm{d} \Gamma
$$

where $\mathbf{t}^{E, \beta}=\boldsymbol{\sigma}^{E, \beta} \mathbf{n}^{\beta}$ is the traction computed from the enriched part of the stress on the boundary of patch $\beta$. This transformation to contour integrals over the boundaries of the patches saves a tremendous amount of computer time.

\section{NUMERICAL STUDIES}

In this section several numerical examples are given. In most of the problems, the accuracy of the standard XFEM, AS-XFEM and DG-XFEM will be compared using the relative energy norm

$$
\text { relative energy norm }=\left(\frac{\int_{\Omega}\left(\boldsymbol{\varepsilon}-\boldsymbol{\varepsilon}^{h}\right): \mathbf{C}:\left(\boldsymbol{\varepsilon}-\boldsymbol{\varepsilon}^{h}\right) \mathrm{d} \Omega}{\int_{\Omega} \boldsymbol{\varepsilon}: \mathbf{C}: \boldsymbol{\varepsilon} \mathrm{d} \Omega}\right)^{1 / 2}
$$

where $\varepsilon^{h}$ is the FE solution and $\boldsymbol{\varepsilon}$ is the exact solution.

\subsection{Material interfaces}

Consider a circular domain of radius $R_{e}$ containing a circular inclusion of radius $R$, as shown in Figure 3. Both the inclusion and the bulk materials are elastic with material properties $\lambda_{1}=0.4$, $\mu_{1}=0.4$ and $\lambda_{2}=5.769, \mu_{2}=3.846$, respectively. The domain is subject to displacements $u_{r}=R_{e}$, and $u_{\theta}=0$ along the outer boundary of the domain. The exact solution for this problem in polar coordinates is

$$
\begin{aligned}
u_{r} & =\left[\left(1-\frac{R_{e}^{2}}{R^{2}}\right) a+\frac{R_{e}^{2}}{R^{2}}\right] r, \quad u_{\theta}=0 \\
\varepsilon_{r r} & =\left(1-\frac{R_{e}^{2}}{R^{2}}\right) a+\frac{R_{e}^{2}}{R^{2}}, \quad \varepsilon_{\theta \theta}=\left(1-\frac{R_{e}^{2}}{R^{2}}\right) a+\frac{R_{e}^{2}}{R^{2}}, \quad 0 \leqslant r \leqslant R \\
\varepsilon_{r \theta} & =0 \\
u_{r} & =\left(r-\frac{R_{e}^{2}}{r}\right) a+\frac{R_{e}^{2}}{r}, \quad u_{\theta}=0 \\
\varepsilon_{r r} & =\left(1+\frac{R_{e}^{2}}{r^{2}}\right) a-\frac{R_{e}^{2}}{r^{2}}, \quad \varepsilon_{\theta \theta}=\left(1-\frac{R_{e}^{2}}{r^{2}}\right) a+\frac{R_{e}^{2}}{r^{2}}, \quad R \leqslant r \leqslant R_{e} \\
\varepsilon_{r \theta} & =0
\end{aligned}
$$

where $r$ is the distance from the centre of the inclusion and

$$
a=\frac{\left(\lambda_{1}+\mu_{1}+\mu_{2}\right)^{2}}{\left(\lambda_{2}+\mu_{2}\right) R^{2}+\left(\lambda_{1}+\mu_{1}\right)^{2}-R^{2}+\mu_{2}^{2}}
$$




\section{R. GRACIE, H. WANG AND T. BELYTSCHKO}
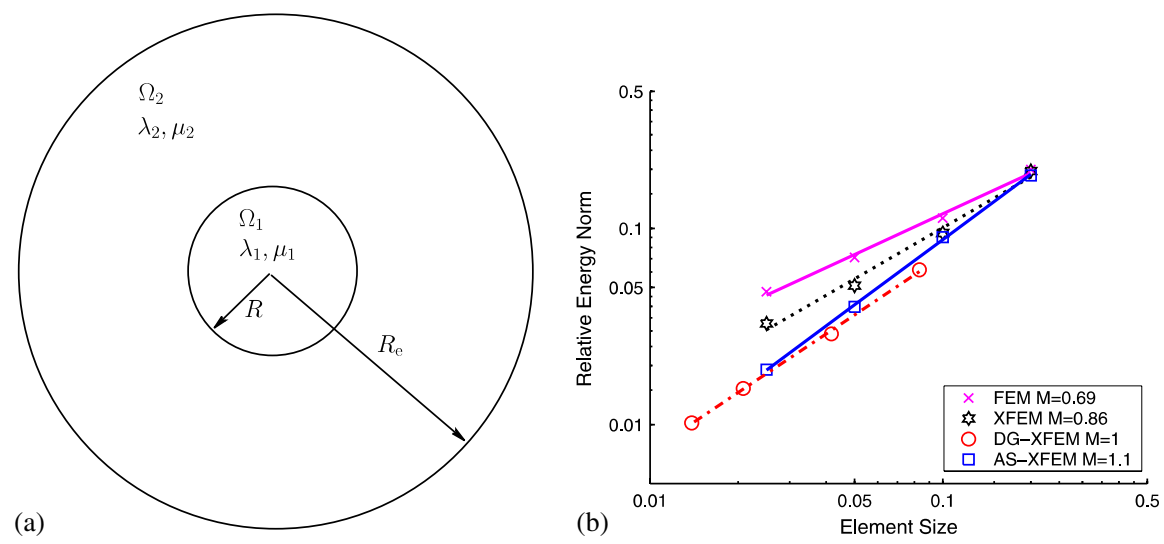

Figure 3. (a) Notation for the problem of a circular domain with a circular inclusion. (b) Convergence of the energy norm for the patch-based discontinuous Galerkin XFEM (DG-XFEM), XFEM with assumed strain blending elements (AS-XFEM), the standard XFEM and the FEM. $M$ is the rate of convergence obtained by linear regression.

The strain across the material interfaces is discontinuous. The weak discontinuity, wedge enrichment developed in Belytschko et al. [29] and Sukumar et al. [9], is used. It is

$$
\Psi(\mathbf{x})=|f(\mathbf{x})|
$$

where $f(\mathbf{x})=0$ defines the location of the interface. This problem was previously solved in Chessa et al. [10] with an AS formulation in the blending elements. The problem is solved by the patchbased DG-XFEM with constant strain triangular elements and a penalty parameter $\alpha=10^{4}$ and by AS-XFEM with bilinear elements. Figure 3(b) shows the convergence of the energy norm relative to element size for the standard XFEM with bilinear elements, the DG-XFEM and the AS-XFEM. We see that the accuracy of DG-XFEM and AS-XFEM is similar and superior to the standard XFEM. The convergence rate of both DG-XFEM and AS-XFEM is optimal, while the standard XFEM is slightly suboptimal, $O\left(h^{0.86}\right)$. FEM gives the poorest accuracy and the least optimal rate of convergence, $O\left(h^{0.70}\right)$.

\subsection{Problems with sources}

In the next two examples, enrichment is added to better approximate the response to body forces. In these examples, DG-XFEM computations are performed with $\alpha=10$.

7.2.1. One-dimensional Laplace equation with harmonic source. In this example we consider a one-dimensional bar, $x \in[0,1]$, of unit stiffness subject to a harmonic body force $b$. The displacements at the ends of the bar are constrained to be zero. The problem is governed by the onedimensional Laplace equation

$$
\nabla^{2} u+b=0
$$


The body force is

$$
b= \begin{cases}0, & x<0.25 \\ \sum_{i=1}^{3} A_{i} \cos \left(k_{i} x\right), & 0.25 \leqslant x \leqslant 0.75 \\ 0, & x>0.75\end{cases}
$$

where $k=[2 \pi, 6 \pi, 18 \pi]$ and $A=[-1,10,50]$. The solution to this problems is

$$
\phi(x)= \begin{cases}a_{1} x, & x<0.25 \\ a_{2}+\sum_{i=1}^{3} \frac{A_{i}}{k_{i}^{2}} \cos \left(k_{i} x\right), & 0.25 \leqslant x \leqslant 0.75 \\ a_{3} x+a_{1}, & x>0.75\end{cases}
$$

where

$$
a_{1}=-\sum_{i=1}^{3} \frac{A_{i}}{k_{i}} \sin \left(k_{i} / 4\right), \quad a_{2}=\frac{a_{1}}{4}-\sum_{i=1}^{3} \frac{A_{i}}{k_{i}^{2}} \cos \left(3 k_{i} / 4\right), \quad a_{3}=-\sum_{i=1}^{3} \frac{A_{i}}{k_{i}} \sin \left(3 k_{i} / 4\right)
$$

We use the enrichment function

$$
\Psi(x)=\phi(x)
$$

In the patch-based DG-XFEM computations, the domain is decomposed into three patches, $\Omega_{\beta}^{P}$, $\beta=1-3$. The domains of patches $\Omega_{1}^{P}, \Omega_{2}^{P}$ and $\Omega_{3}^{P}$ are $0 \leqslant x \leqslant 0.25,0.25 \leqslant x \leqslant 0.75$ and $0.75 \leqslant x \leqslant 1$, respectively. We enrich patch $\Omega_{2}^{P}$ by (62) and patches $\Omega_{1}^{P}$ and $\Omega_{3}^{P}$ are unenriched. In the AS-XFEM and the standard XFEM computations all nodes such that $0.25 \leqslant x_{I} \leqslant 0.75$ are enriched by (62).

This problem was solved in Chessa et al. [10] with an AS formulation for the blending elements, and it was shown that AS-XFEM gives the exact result. Figure 4 shows the displacement and strain fields from the standard XFEM and DG-XFEM with a uniform mesh of eight elements. We can see that the solution from the standard XFEM is not accurate in the blending elements, i.e. $0.125<x<0.25$ and $0.75<x<0.875$. In contrast, DG-XFEM, like the AS formulation, gives the exact result to within machine precision. We note that the pollution of the standard XFEM solution by the parasitic terms is clearly visible in both the displacement and strain fields (Figures 4(a) and $4(\mathrm{~b})$ ).

7.2.2. Two-dimensional elastic domain with a singular body force. Consider an $L \times L$ domain with $L=1$. The origin of the coordinate system is located at the centre of the domain. The material is elastic with modulus $E=1$ and the Poisson ratio $v=0.3$, and is in plane strain. The boundary conditions are

$$
\begin{aligned}
& \left.\mathbf{u}\right|_{x=0}=\mathbf{u}^{s},\left.\quad \mathbf{u}\right|_{y=0}=\mathbf{u}^{s} \\
& \left.\mathbf{t}\right|_{x=L}=\sigma\left(\mathbf{u}^{s}\right) \cdot \mathbf{n},\left.\quad \mathbf{t}\right|_{y=L}=\sigma\left(\mathbf{u}^{s}\right) \cdot \mathbf{n}
\end{aligned}
$$



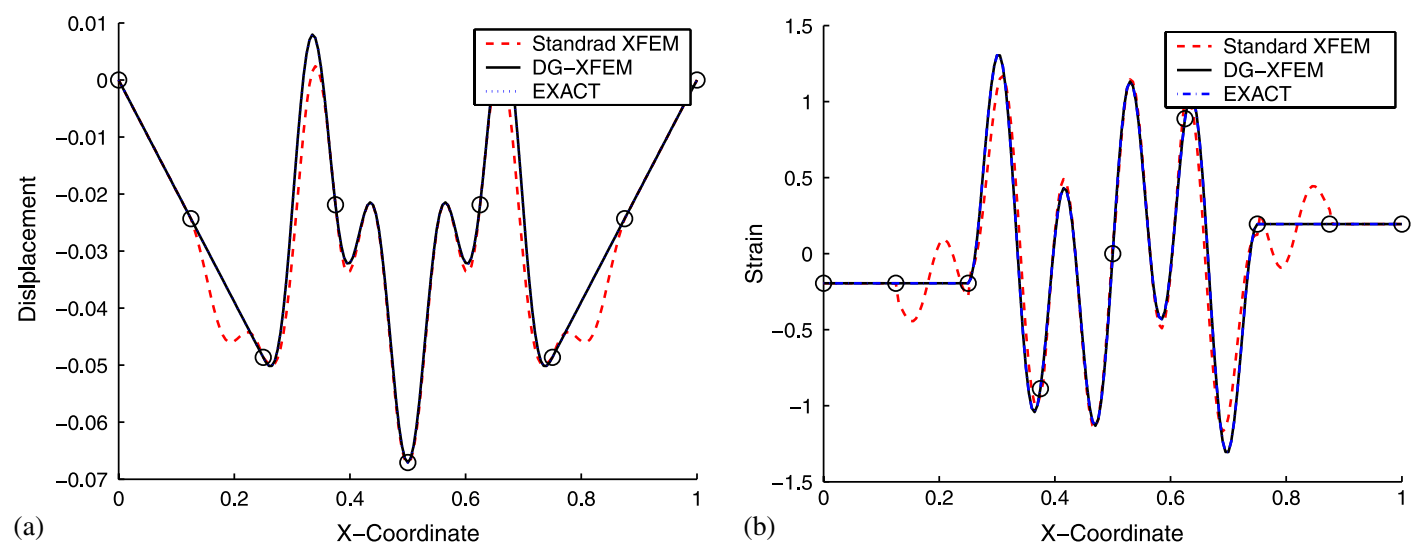

Figure 4. (a) Comparison of the results from the standard XFEM and the patch-based discontinuous Galerkin XFEM (DG-XFEM) for the harmonic source problem: (a) the displacement field and (b) the strain field.

where $\mathbf{n}$ is the normal to the boundary, and $\mathbf{u}^{s}$ will be defined below. The body is subject to a body force, which is chosen so that equilibrium is satisfied:

$$
\mathbf{b}=\left[\begin{array}{c}
-\frac{E\left(y-y_{0}\right)\left(3\left(x-x_{0}-\left(y-y_{0}\right)\right)+2 v r^{2}\right)}{8\left(2 v^{2}+v-1\right) r^{7 / 2}} \\
-\frac{E\left(3\left(x-x_{0}\right)\left(y-y_{0}-\left(x-x_{0}\right)\right)+2 v r^{2}\right)}{8\left(2 v^{2}+v-1\right) r^{7 / 2}}
\end{array}\right]
$$

where $r=\sqrt{\left(x-x_{0}\right)^{2}+\left(y-y_{0}\right)^{2}}$. The displacement field is given by

$$
\mathbf{u}^{s}=\left[\begin{array}{c}
\sqrt{r} \\
\sqrt{r}
\end{array}\right]
$$

We adopt the enrichment function $\Psi=\mathbf{u}^{s}$. The domain is discretized uniformly with bilinear elements. We use the same element topology for both the standard XFEM and DG-XFEM computations. In the standard XFEM computations, the nodes within a distance of $r_{0}=0.2$ from the singularity are enriched. In the patch-based DG-XFEM computations, the domain is decomposed into two patches-one which is enriched and the other which is not enriched. The enriched patch consists of all elements for which all the nodes of the element are within a distance $r_{0}=0.2$ from the singularity.

Figure 5(a) shows the shear strain along the line $y=0.5$ obtained by the standard XFEM and the DG-XFEM for a mesh of $11 \times 11$ bilinear elements. We note that in the blending region, $0.63 \leqslant x \leqslant 0.74$, the standard XFEM solution differs substantially from DG-XFEM and the exact solutions. The error in the blending element also pollutes the solution near the singularity, i.e. in elements with all nodes enriched.

In Figures 5(b) and (c) the convergence of the energy norm is shown for the standard XFEM and for both the patch-based and element-based DG-XFEM methods. Here we see that all methods converge at the optimum rate. For a given mesh both the element-based and patch-based DG-XFEM 

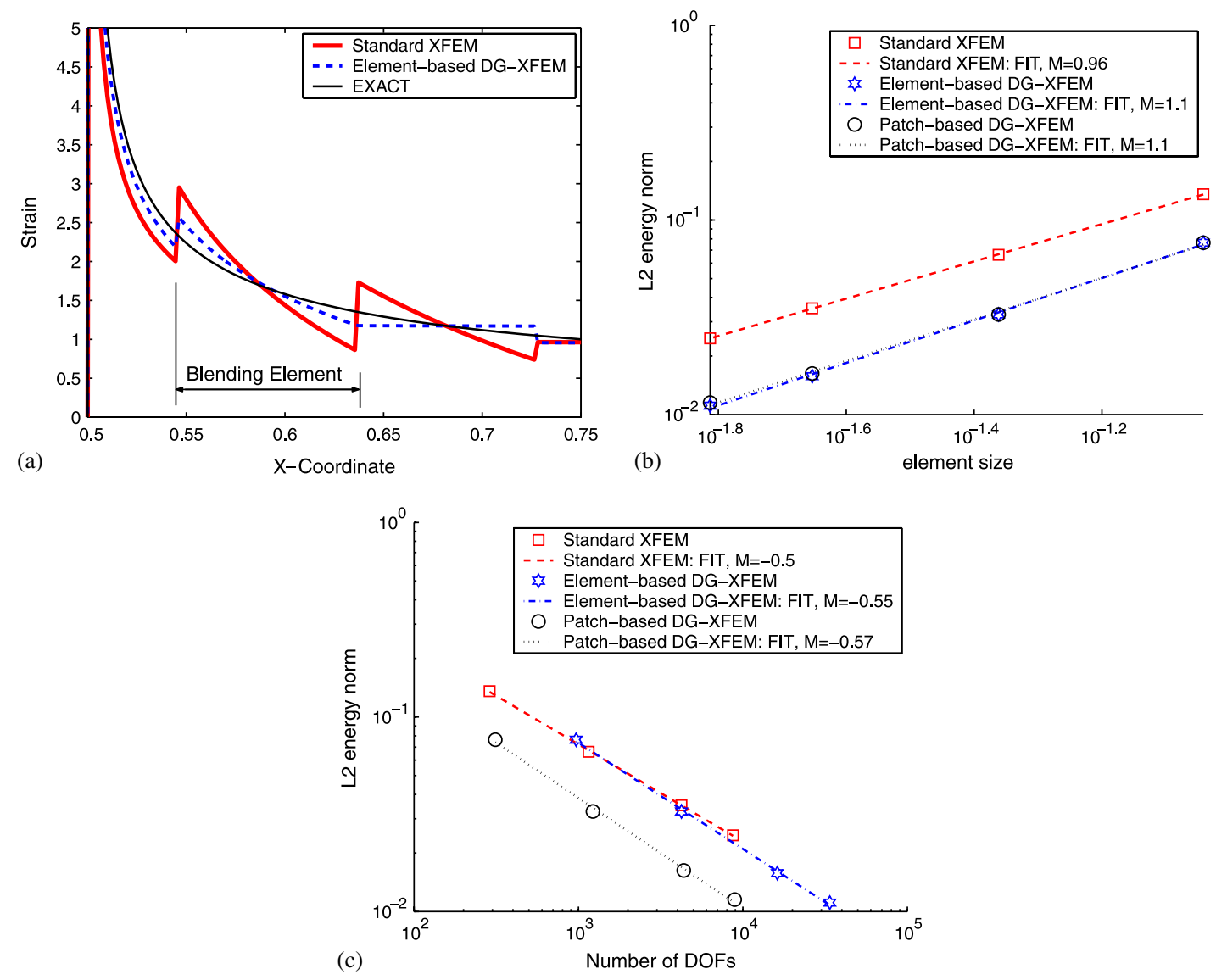

Figure 5. (a) Comparison of the shear strain, plotted along the line $y=0$, by the standard XFEM, the element-based discontinuous Galerkin XFEM (DG-XFEM) and the exact solution (EXACT), for an elastic body with a singular body force. (b) Convergence of the energy norm versus element size and (c) versus number of degrees of freedom (DOF). $M$ is the rate of convergence.

methods are significantly more accurate than the standard XFEM. For a given number of degrees of freedom, the element-based DG-XFEM and the standard XFEM have similar accuracies, while the patch-based DG-XFEM is substantially more accurate than either of the other two methods.

\subsection{Cracks and dislocations}

Next we examine crack and edge dislocation problems. These problems are particularly challenging for the standard FEM since in addition to discontinuities in the displacement field, they include the singular stress fields. We will apply the DG method with bilinear elements and a penalty parameter $\alpha=100 E$, where $E$ is the elastic modulus of the material.

7.3.1. Mode I crack. Consider an infinite body with elastic modulus, $E=1000$ and the Poisson ratio, $v=0.3$ with a centre crack of length $2 a=10$, loaded by a remote stress $\sigma_{\infty}=1$ normal to the 

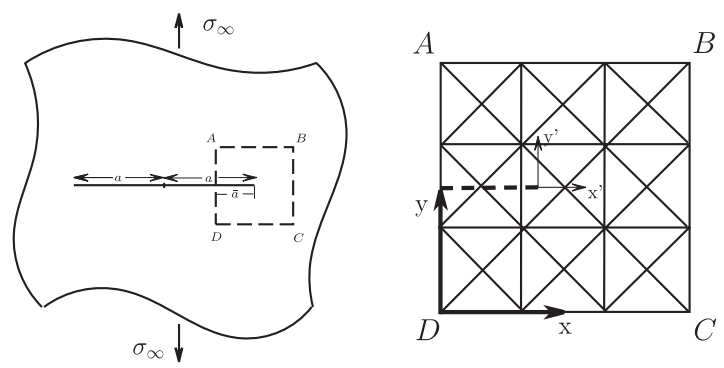

Figure 6. Illustration of the infinite body with a Mode I crack and the subdomain $A B C D$ that is approximated with XFEM.

crack. The FE model $A B C D$ is of size $1 \times 1$ with $\bar{a}=0.55$, as shown in Figure 6 . The point $D$ is chosen as the origin of the coordinate system; the crack tip is at $(\bar{a}, 0.5)$. Displacement boundary conditions are applied to the boundary of $A B C D$ corresponding to the asymptotic solution for a crack of length $2 a$ in an infinite body.

The displacement field of the asymptotic plain strain solution is

$$
\begin{aligned}
& u_{x}=\frac{2(1+v)}{\sqrt{2 \pi}} \frac{\sqrt{r} K_{I}}{E} \cos \frac{\theta}{2}\left(2-2 v-\cos ^{2} \frac{\theta}{2}\right) \\
& u_{y}=\frac{2(1+v)}{\sqrt{2 \pi}} \frac{\sqrt{r} K_{I}}{E} \sin \frac{\theta}{2}\left(2-2 v-\cos ^{2} \frac{\theta}{2}\right)
\end{aligned}
$$

where $r$ and $\theta$ are defined as in Figure 2 and the Mode I stress intensity factor (SIF) is given by $K_{I}=\sigma_{\infty} \sqrt{\pi a}$.

We will first compare the standard XFEM with the AS-XFEM. We use enrichments (30) and (31); all nodes within a radius of $r_{0}=0.15$ from the crack tip are enriched by (30) while all nodes with support cut by the crack but not enriched by (30) are enriched by (31). SIFs are calculated using the domain form of the $J$-integral of Moran and Shih [32] with a circular domain of radius 0.2 .

One question that arises in AS-XFEM is the choice of the strain approximation for postprocessing. The strain can be computed either from (24) or by taking the symmetric gradient of the displacement. In Figure 7(a), the normal stresses along the crack $(y=0.5, \theta=0)$ obtained from both the displacement gradient and the strain approximations are shown for a cross-triangular mesh as in Figure 6 with $7 \times 7$ cells (196 elements). The two are identical except in the blending elements, $0.36<x<0.43$ and $0.64<x<0.74$. The normal stresses obtained from the strain approximation are better ahead of the crack tip than those obtained from the displacement gradient. The energy norm is also smaller when the stresses are derived from (24); hence, we consider the stress from (24) as more effective and we will use it in our comparisons with the classical XFEM and for the calculation of SIFs.

Figure 7(b) compares the normal stresses, $\sigma_{y y}$, from the standard XFEM and the AS-XFEM with constant strain triangular elements. We can see that AS-XFEM more accurately satisfies the traction-free boundary conditions on the crack faces. As a result, the assumption of traction-free crack surfaces made in the calculation of SIFs by the $J$-integral or by the interaction integrals is adhered to more closely. 

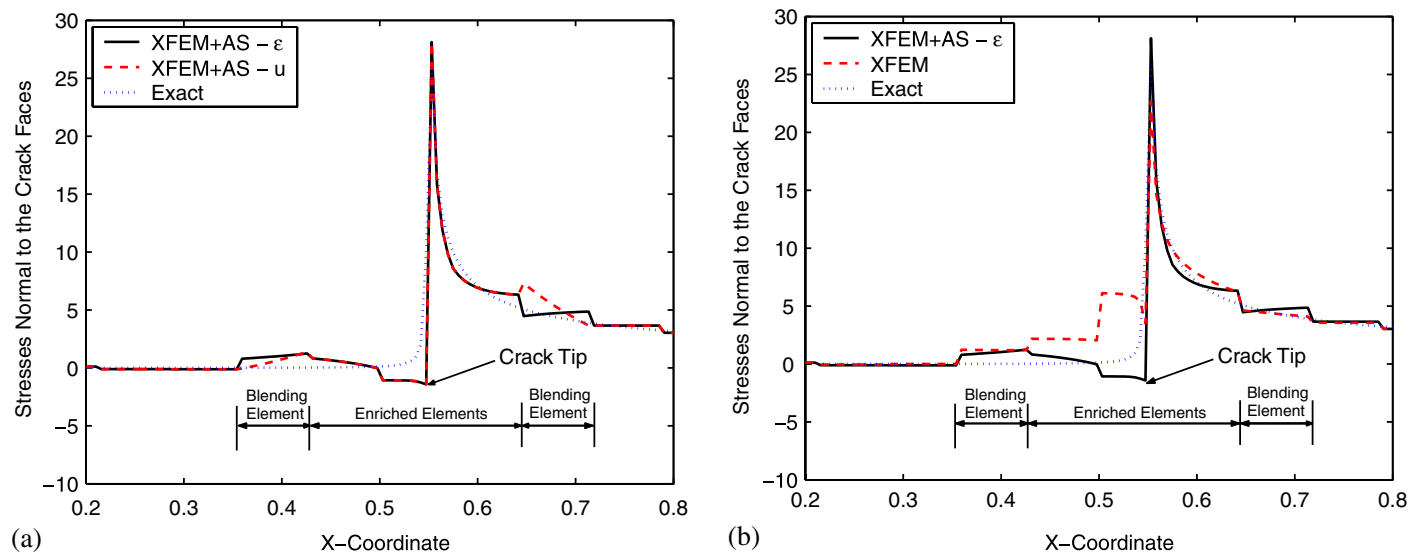

Figure 7. Results for the normal stresses along the line defining the crack surface for the infinite plate problem. (a) Comparison of the normal stress, $\sigma_{y y}$, computed form both the strain approximation and the displacement gradient. $\sigma_{y y}\left(\nabla_{s} \mathbf{u}\right)$ and $\sigma_{y y}(\overline{\boldsymbol{\varepsilon}})$ are denoted as XFEM-AS- $u$ and XFEM-AS- $\varepsilon$, respectively. (b) The normal stresses from the XFEM with AS blending elements are compared with the stresses from the classical XFEM.
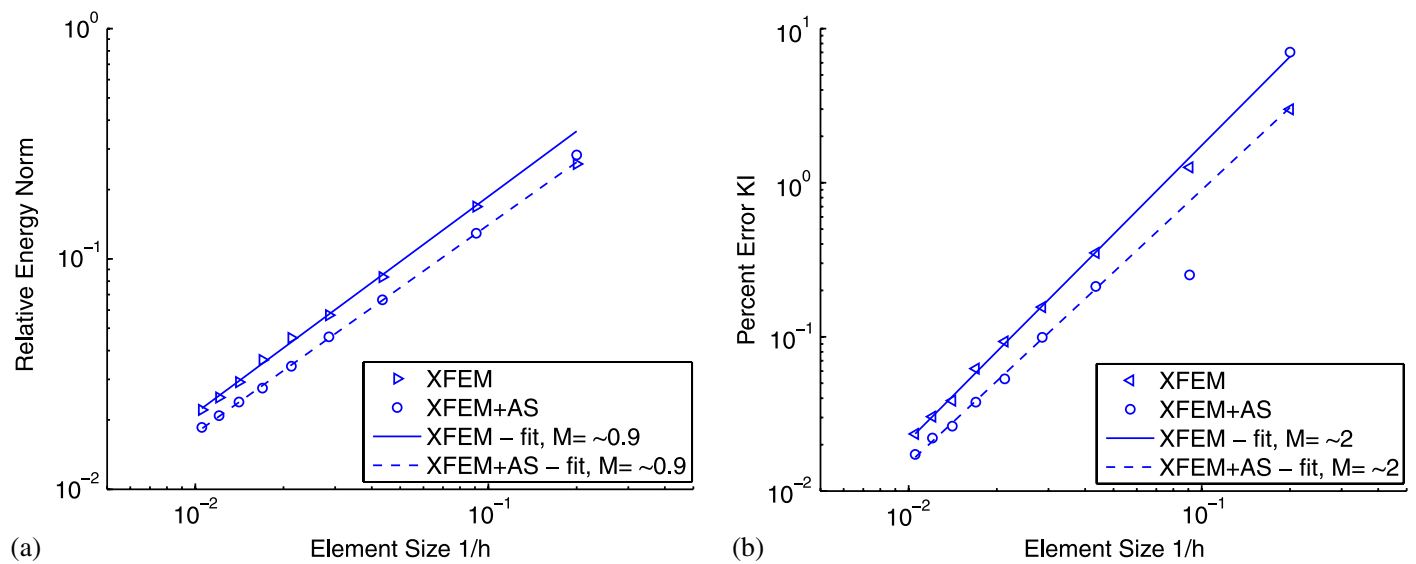

Figure 8. Convergence plots for the cracked infinite plate problem relative to element size for the standard XFEM and for XFEM with assumed strain blending elements (AS-XFEM). (a) The relative energy norm and (b) Mode I stress intensity factor. $M$ is the rate of convergence; 'fit' indicates a linear regression fit to the data points.

Convergence of the relative energy norm is shown in Figure 8(a). Here we can see that ASXFEM achieves the optimal convergence rate of $O(h)$ in the energy norm and that its accuracy is improved over the standard XFEM. Figure 8(b) shows the convergence of the Mode I SIF for ASXFEM and the standard XFEM. Here again AS-XFEM increases the accuracy while maintaining the optimal convergence rate of $O\left(h^{2}\right)$. It should be noted that the SIFs were calculated with the assumption that the crack surface traction is zero. 


\section{R. GRACIE, H. WANG AND T. BELYTSCHKO}

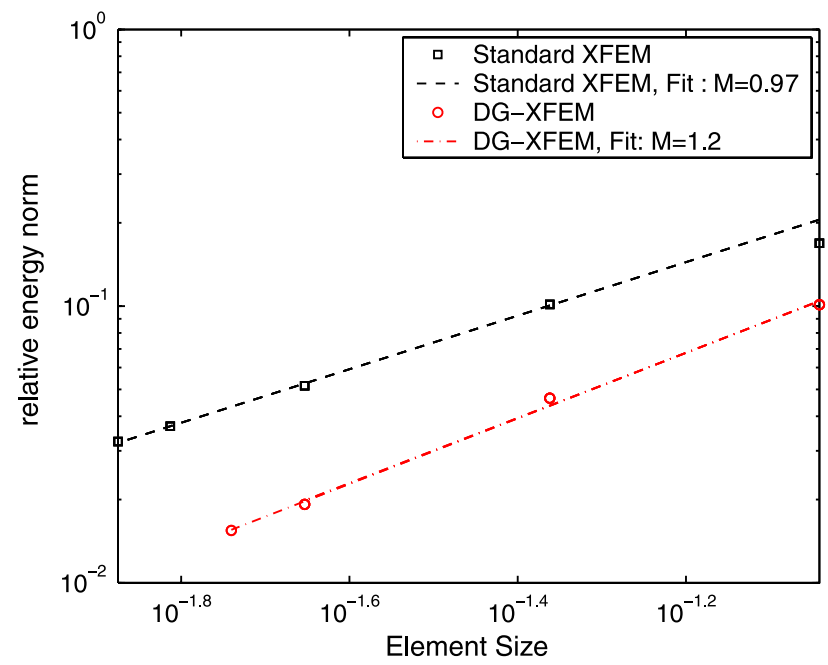

Figure 9. Comparison of the convergence of the energy norm for the standard XFEM and the discontinuous Galerkin XFEM (DG-XFEM) for the cracked infinite plate problem. $M$ is the rate of convergence; 'fit' indicates a linear regression fit to the data points.

Next we compare the standard XFEM with DG-XFEM. We solve the same problem but using the singular crack tip enrichment of Liu et al. [33]. We use a uniform discretization of bilinear elements. The same element topology is used for the computations of both the standard XFEM and the DG-XFEM.

In the standard XFEM computations, the enriched nodes are as previously defined. In the patchbased DG-XFEM computations, the domain is decomposed into three patches-the first is enriched by the crack tip enrichment of Liu et al. [33], the second is enriched by the jump function (31) and the third is unenriched. The first patch is composed of any element such that all nodes of the element are within a distance of 0.15 from the crack tip. The second patch is composed of elements that are cut by the crack but are not in the first patch and the third patch is composed of all elements not in the first two patches.

Figure 9 shows the convergence of the energy norm for uniform meshes of bilinear elements. The standard XFEM converges optimally while DG-XFEM is slightly superconvergent. DG-XFEM is seen to be more accurate than the standard XFEM with respect to element size.

7.3.2. Edge dislocation. Consider an edge dislocation in an infinite elastic domain with elastic modulus $E=10^{5}$ and the Poisson ratio $v=0.3$. We model an $L \times L$ finite domain, with $L=1$. The origin is located at the bottom left corner of the domain; the core is located at $x=y=0.5$. The glide plane of the dislocation is along the line $y=0.5$, and the Burgers vector is in the $x$-direction with a magnitude $b=10^{-3}$. The solution is given by Equation (36). We apply displacement boundary conditions equivalent to the exact solution (36) on the four boundaries and use the enrichment functions (36) and (37).

A uniform element topology is used for all simulations. In the standard XFEM computations, we enrich all nodes within a distances of 0.2 from the core with (36). Any node that is not enriched by (36) but with support cut by the glide plane is enriched by (37). In the patch-based DG-XFEM 

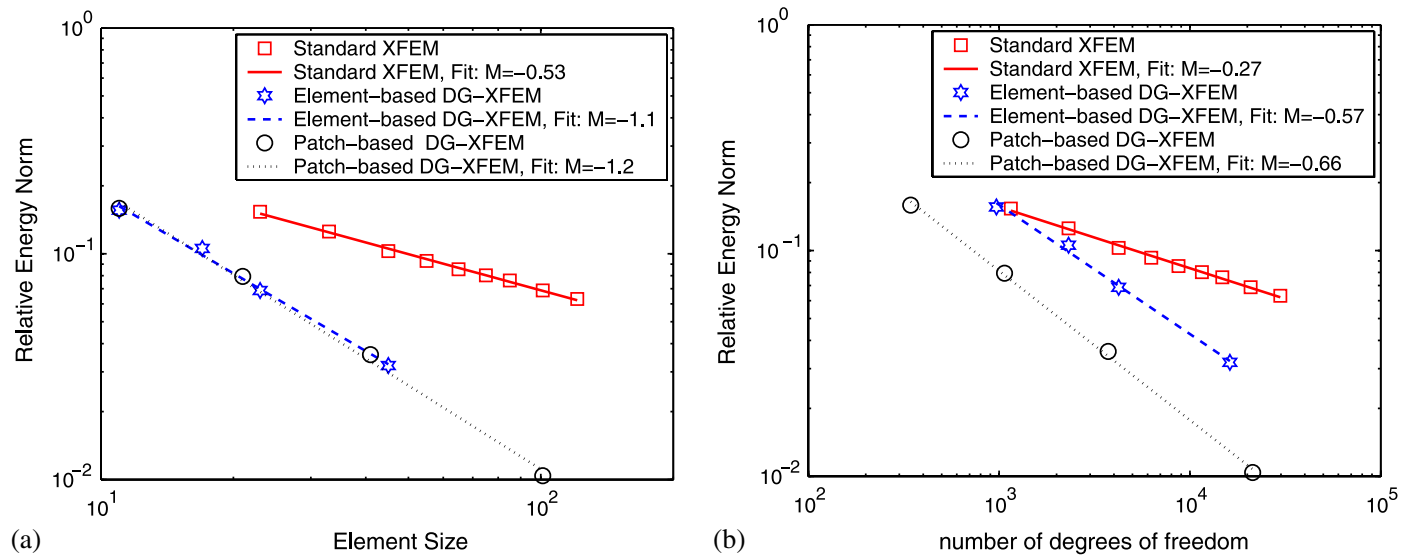

Figure 10. Convergence of the relative energy norm obtained with the standard XFEM, the element-based discontinuous Galerkin XFEM (DG-XFEM) and the patch-based DG-XFEM for the problem of an edge dislocation in an infinite domain. $M$ is the rate of convergence; 'fit' indicates a linear regression fit to the data.

computations, the domain is decomposed into three patches-the first is enriched by the core enrichment functions (36), the second is enriched by the tangent jump function (37) and the third is unenriched. The first patch is composed of any element such that all nodes of the element are within a distance of 0.2 from the core. The second patch is composed of elements that are cut by the glide plane but are not in the first patch and the third patch is composed of all elements not in the first two patches.

The strain energy of the dislocation is infinite at the core; hence, we neglect a region of radius 0.05 about the core when computing the energy norm. The convergence of the energy norm for the standard XFEM, the element-based DG-XFEM and the patch-based DG-XFEM are shown in Figure 10.

In this application, the standard XFEM converges suboptimally in the energy norm, while the DG-XFEM schemes are both slightly superconvergent with respect to element size. From Figure 10(a) we observe that the accuracy of the element-based and patch-based DG-XFEM is almost the same for a given element size, but from Figure 10(b) we see that the patch-based DG-XFEM is significantly more accurate for a given number of degrees of freedom. Both DG-XFEM schemes are more accurate than the standard XFEM for a given number of degrees of freedom. This is in contrast to the previous examples, where the accuracy of the element-based DG-XFEM was similar to that of the standard XFEM for a given number of degrees of freedom.

\section{DISCUSSION AND CONCLUSIONS}

A significant part of the error in local enrichment methods such as the extended finite element method (XFEM) is known to originate in the blending elements, i.e. the partially enriched elements. We have described two discontinuous Galerkin (DG) forms of XFEM (DG-XFEM), which eliminate these blending elements: a patch-based and an element-based form. In the patch-based formulation, 


\section{R. GRACIE, H. WANG AND T. BELYTSCHKO}

the domain is decomposed into non-overlapping patches. Enrichments are applied over these patches and continuity between the patches is enforced with an internal penalty (IP) method. In the element-based form, each element is treated as a patch.

Both DG forms of XFEM have the desirable characteristic that the enrichment is local and that all shape functions form a partition of unity (PU). This is in contrast to the standard XFEM where the PU property of the shape functions that pre-multiply the enrichment functions do not satisfy the PU property everywhere in the domain.

In all problems studied here, DG-XFEM provides excellent results with a modest penalty factor $(10 E-100 E)$. Therefore, the conditioning of the equations is not significantly impaired and iterative methods for the solution of the linear system equations are still effective. In fact, we have observed that the accuracy of DG-XFEM is often reduced when very large penalties are used. This is because the approximation spaces of adjacent patches are often incompatible. When adjacent patches are incompatible, very large penalty terms have the effect of driving the enriched degrees of freedom to zero.

We have also considered the assumed strain (AS-XFEM) approach, in which the error due to blending is reduced by eliminating the parasitic term in the strain approximation of the blending elements. An advantage of AS-XFEM, in comparison with DG-XFEM, is that the additional AS coefficients are solved for at the element level; as a result, it is more easily implemented into standard FE programs. However, the selection of the AS shape functions can be quite difficult. Moreover, the AS shape functions depend on the enrichment; hence, the method has to be reformulated specifically for each enrichment. In contrast, the DG-XFEM implementation is more independent of the enrichments. As a result, incorporation of additional enrichments into an existing DG code is straightforward.

In the modelling of interfaces by the wedge enrichment, it was found that neither the FEM nor the standard XFEM converges optimally, although the accuracy of XFEM is much better than the standard FEM and may be acceptable for many purposes. It was shown that the patch-based DGXFEM and AS-XFEM converge optimally and are more accurate than the FEM and the standard XFEM. The AS-XFEM and DG-XFEM have similar accuracy for a given element size.

Several manufactured solutions were considered. In the one-dimensional problem, the standard XFEM solution deviates significantly from the exact solution, while the patch-based DG-XFEM gives the exact result, as is the case for AS-XFEM.

We also considered manufactured solutions for two-dimensional domains with singular stress fields. Enrichment was added to the approximation to augment the standard FEM approximation near the singularity. It was shown that the standard XFEM and both the element-based and the patch-based DG-XFEM converge optimally in the energy norm. For a given element size, both DG-XFEM forms have similar accuracy and are more accurate than the standard XFEM. However, for a given number of degrees of freedom, the standard XFEM and the element-based DG-XFEM have similar accuracies, while the patch-based DG-XFEM is significantly more accurate than the other two methods.

For elastic cracks under Mode I loading, it was shown that for constant stress triangular elements, the standard XFEM does not accurately satisfy the traction-free boundary conditions along the crack faces. The AS-XFEM was shown to improve the approximation of the traction-free boundary conditions and to improve the accuracy as compared with the standard XFEM. It was shown that the standard XFEM, AS-XFEM and both the element-based and the patch-based DG-XFEM all converge optimally in the energy norm. For a given element size, AS-XFEM and both DG-XFEM forms have similar accuracies and are significantly more accurate than the standard XFEM. 
In dislocation modelling, in contrast to the other numerical problems studied, the enriched degrees are prescribed. As a result, the effect of the enrichment appears as nodal forces on the righthand side of the discrete equations. For the DG-XFEM, we developed a particularly efficient form of the nodal force equations. The standard domain integrals over each element were transformed into contour integrals over the boundaries of the patches. This contour form is significantly more computationally efficient.

It was shown that for dislocations, the convergence of the energy error is suboptimal for the standard XFEM but was optimal for both DG-XFEM methods. In addition, both DG-XFEM methods are more accurate than the standard XFEM for a given number of degrees of freedom and for a given element size. The patch-based and the element-based DG-XFEM have similar accuracies for a given element size; however, the patch-based DG-XFEM is more accurate for a given number of degrees of freedom.

When DG-XFEM is used for dislocation dynamics, the element-based form can be advantageous over the patch-based form because the same stiffness matrix can be used for an entire simulation. By contrast the stiffness matrices for AS-XFEM and the patch-based DG-XFEM change when the enrichment patches are moved.

The accuracy of XFEM in dislocation modelling is more severely impaired by blending than in crack and weak discontinuity models because the enriched degrees of freedom are prescribed. This reduces the flexibility of the approximation to correct for the parasitic terms. The accuracy of the standard XFEM can be slightly improved by not prescribing the singular enrichment degrees of freedom of the nodes at the edge of the enrichment domain; however, the accuracy is still much less than that of DG-XFEM.

We have found that the element-based DG-XFEM is easier to implement because the boundaries of the enrichment subdomains do not have to be identified. This is especially significant when the enrichments evolve during a simulation. We have found that when only a single feature requires enrichment, as in the problems studied here, the element-based DG-XFEM generally involves significantly more degrees of freedom than the patch-based DG-XFEM and that the accuracy of the patch based is superior to that of the element-based DG-XFEM for a given number of degrees of freedom. Since the accuracy of the element-based and the patch based DG-XFEM are similar for a given element size, we have concluded that the application of DG between two unenriched patches neither impairs nor improves the accuracy of the simulation.

When the boundaries of the enrichments are restricted to a small set of element edges it will generally be desirable to use a patch-based DG-XFEM rather than an element-based DG-XFEM. However, when many enrichments are used, the number of element edges where the DG penalty term is applied will approach the total number of element edges in the domain. Therefore, the performance of the patch-based DG-XFEM and element-based DG-XFEM for a given number of degrees of freedom would be similar. In such situations the adoption of the element-based DG-XFEM will be attractive because of its ease of implementation.

From the numerical studies conducted, we observe that the degree to which blending affects the accuracy and the convergence rate of the XFEM depends greatly on the enrichment. The DGXFEM is most effective when the standard XFEM converges suboptimally, as in the modelling of intra-element material interfaces and dislocations.

The accuracy of AS-XFEM and DG-XFEM is generally similar for a given element size; in addition, both methods have been shown to converge optimally for all enrichments considered. Therefore, the choice of which method to implement is governed by ease of adoption to a given application. Clearly, both methods eliminate the spurious effects arising from the blending elements 


\section{R. GRACIE, H. WANG AND T. BELYTSCHKO}

in the XFEMs. Since the locality of enrichment is crucial for efficiency, both the AS and DG methods are of substantial practical interest.

\section{ACKNOWLEDGEMENTS}

The support from the Office of Naval Research under grant N00014-06-1-380 and the Army Research Office under grant W911NF-05-1-0049 and the Natural Sciences and Engineering Research Council under a Canada Graduate Scholarship are gratefully acknowledged.

\section{REFERENCES}

1. Belytschko T, Black T. Elastic crack growth in finite elements with minimal remeshing. International Journal for Numerical Methods in Engineering 1999; 45:601-620.

2. Moës N, Dolbow J, Belytschko T. A finite element method for crack growth without remeshing. International Journal for Numerical Methods in Engineering 1999; 46:131-150.

3. Melenk JM, Babuška I. The partition of unity finite element method: basic theory and applications. Computer Methods in Applied Mechanics and Engineering 1996; 139:290-314.

4. Song J-H, Areias PMA, Belytschko T. A method for dynamic crack and shear band propagating with phantom nodes. International Journal for Numerical Methods in Engineering 2006; 67(6):868-893.

5. Asferg JL, Poulsen PN, Nielsen LO. A consistent partly cracked XFEM element for cohesive crack growth. International Journal for Numerical Methods in Engineering 2007; 72(4):464-485.

6. Simone A, Duarte CA, Van der Giessen E. A generalized finite element method for polycrystals with discontinuous grain boundaries. International Journal for Numerical Methods in Engineering 2006; 67:1122-1145.

7. Ventura G, Moran B, Belytschko T. Dislocations by partition of unity. International Journal for Numerical Methods in Engineering 2005; 62(11):1463-1487.

8. Gracie R, Ventura G, Belytschko T. A new fast method for dislocations based on interior discontinuities. International Journal for Numerical Methods in Engineering 2007; 69:423-441.

9. Sukumar N, Chopp DL, Moës N, Belytschko T. Modeling holes and inclusions by level sets in the extended finite-element method. Computer Methods in Applied Mechanics and Engineering 2001; 190:6183-6200.

10. Chessa J, Wang HW, Belytschko T. On the construction of blending elements for local partition of unity enriched finite elements. International Journal for Numerical Methods in Engineering 2003; 57:1015-1038.

11. Legay A, Wang HW, Belytschko T. Strong and weak arbitrary discontinuities in spectral finite elements. International Journal for Numerical Methods in Engineering 2005; 64(8):991-1008.

12. Reed WH, Hill TR. Triangular mesh methods for the neutron transport equation. Los Alamos Scientific Laboratory Report LA-UR-73-479, 1973.

13. Bassi F, Rebay S. High-order accurate discontinuous finite element solution of the 2D Euler equations. Journal of Computational Physics 1997; 138(2):251-285.

14. Cockburn B, Shu C-W. The local discontinuous Galerkin method for time-dependent convection-diffusion systems. SIAM Journal on Numerical Analysis 1998; 35(6):2440-2463.

15. Baumann CE, Oden JT. A discontinuous hp finite element method for the Euler and Navier-Stokes equations. International Journal for Numerical Methods in Fluids 1999; 31(1):79-95.

16. Wheeler M. An elliptic collocation-finite element method with interior penalties. SIAM Journal on Numerical Analysis 1978; 15:152-161.

17. Arnold DN. An interior penalty finite element method with discontinuous elements. SIAM Journal on Numerical Analysis 1982; 19(4):742-760.

18. Arnold DN, Brezzi F, Cockburn B, Marini LD. Unified analysis of discontinuous Galerkin methods for elliptic problems. SIAM Journal on Numerical Analysis 2002; 39(5):1749-1779.

19. Pfeiffer AMD. Discontinuous Galerkin methods for elastodynamics. Master's Thesis, Delft University of Technology, 2005.

20. Farhat C, Harari I, Franca LP. The discontinuous enrichment method. Computer Methods in Applied Mechanics and Engineering 2001; 190(48):6455-6479.

21. Duarte AVC, do Carmo EGD, Rochinha FA. Consistent discontinuous finite elements in elastodynamics. Computer Methods in Applied Mechanics and Engineering 2000; 190(1):193-223. 


\section{BLENDING IN XFEM BY DG AND AS METHODS}

22. Laborde P, Pommier J, Renard Y, Salaun M. High-order extended finite element method for cracked domains. International Journal for Numerical Methods in Engineering 2005; 64(3):354-381.

23. Farhat C, Harari I, Hetmaniuk U. A discontinuous Galerkin method with Lagrange multipliers for the solution of Helmholtz problems in the mid-frequency regime. Computer Methods in Applied Mechanics and Engineering 2003; 192(11):1389-1419.

24. Simo JC, Rifai MS. A class of mixed assumed strain methods and the method of incompatible modes. International Journal for Numerical Methods in Engineering 1990; 29:1595-1638.

25. Stolarski H, Belytschko T. Limitation principles for mixed finite elements based on the Hu-Washizu variational formulation. Computer Methods in Applied Mechanics and Engineering 1987; 60(2):195-216.

26. Belytschko T, Liu WK, Moran B. Nonlinear Finite Elements for Continua and Structures. Wiley: New York, 2000.

27. Fleming M, Chu YA, Moran B, Belytschko T. Enriched element-free Galerkin methods for crack tip fields. International Journal for Numerical Methods in Engineering 1997; 40(8):1483-1504.

28. Stolarska M, Chopp DL, Moës N, Belytschko T. Modelling crack growth by level sets in the extended finite element method. International Journal for Numerical Methods in Engineering 2001; 51(8):943-960.

29. Belytschko T, Moës N, Usui S, Parimi C. Arbitrary discontinuities in finite elements. International Journal for Numerical Methods in Engineering 2001; 50:993-1013.

30. Belytschko T, Gracie R. On XFEM applications to dislocations in problems with interfaces. International Journal of Plasticity 2007; 23(10-11):1721-1738.

31. Gracie R, Oswald J, Belytschko T. On a new extended finite element method for dislocations: core enrichments. Journal of Mechanics and Physics of Solids 2007; DOI: 10.1016/j.jmps.2007.07.010.

32. Moran B, Shih CF. A general treatment of crack tip contour integrals. International Journal of Fracture 1987; 35(4):79-109.

33. Liu XY, Xiao QN, Karihaloo BL. XFEM for direct evaluation of mixed mode SIFs in homogeneous and bi-materials. International Journal for Numerical Methods in Engineering 2004; 59:1103-1118. 